\title{
Machine learning for reconstruction of highly porous structures from FIB-SEM nano-tomographic data ${ }^{\star}$
}

\author{
Chiara Fend $^{1,2}$, Ali Moghiseh ${ }^{2}$, Claudia Redenbach ${ }^{1}$, and Katja Schladitz ${ }^{2}$ \\ 1 Department of Mathematics, University of Kaiserslautern, Germany \\ redenbach@mathematik.uni-kl.de \\ 2 Fraunhofer-Institut für Techno- und Wirtschaftsmathematik, Kaiserslautern, \\ Germany \\ \{chiara.fend, ali.moghiseh, katja.schladitz\}@itwm.fraunhofer.de
}

\begin{abstract}
Reconstruction of highly porous structures from FIB-SEM image stacks is a difficult segmentation task. Supervised machine learning approaches demand large amounts of labeled data for training, that are hard to get in this case. A way to circumvent this problem is to train on simulated images. Here, we report on segmentation results derived by training a convolutional neural network solely on simulated FIB-SEM image stacks of realizations of a variety of stochastic geometry models.
\end{abstract}

Keywords: U-net 3D, shine through artifacts, SEM simulation, Boolean model, random packing, Altendorf-Jeulin model, Cox-Boolean model, deep learning

\section{Introduction}

The micro-structure of materials influences their macroscopic properties decisively. 3D images of the micro-structure yield deeper insight into the microstructure's geometric features and can be used for numeric simulations of materials properties like mechanical strength, filtration properties or thermal conductivity. Combined with stochastic geometry models $[10,16]$, they are the basis for optimizing the micro-structure - so-called virtual material design.

Serial slicing by a focused ion beam (FIB) and subsequent imaging by scanning electron microscopy (SEM) is a versatile source for high-quality $3 \mathrm{D}$ images of materials structures at the scale of $3-200 \mathrm{~nm}$. For highly porous structures however, reconstruction of the 3D structure from the SEM image stack is hampered by the solid structure from deeper layers being visible through the pores. These so-called shine through artifacts [12] cause the typical tails visible in the planes orthogonal to the SEM imaging plane, see Figure 1(c). These artifacts featuring the same gray values as the true foreground in the current slice, thresholding methods fail to segment the solid structure properly. Several algorithms

\footnotetext{
* Supported by German Federal Ministry of Education and Research, project 03VP00491/5, and Fraunhofer FLAGSHIP PROJECT ML4P.
} 


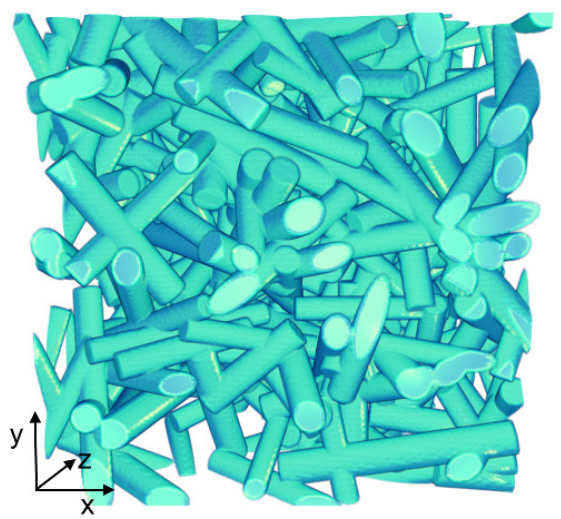

(a) Volume rendering

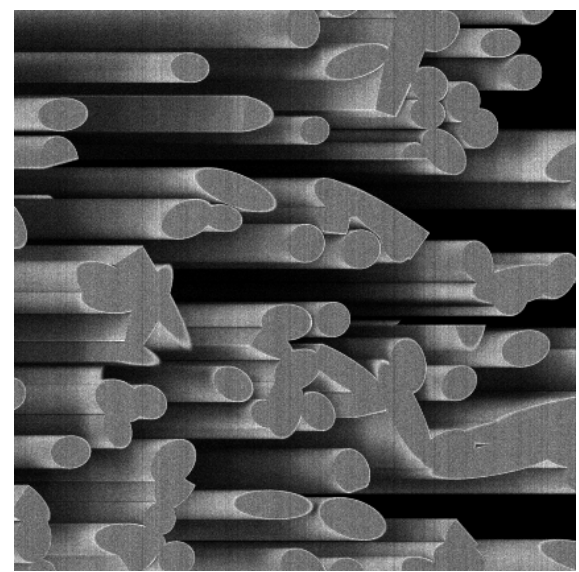

(c) Side (xz) view of simulated FIB-SEM stack

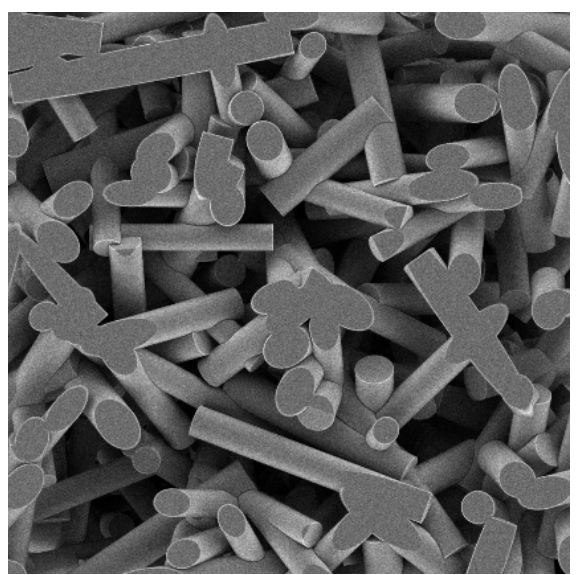

(b) Simulated BSE, xy-slice

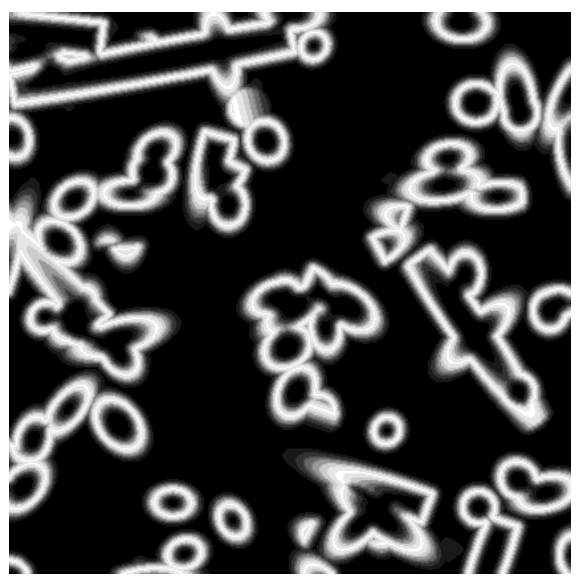

(d) Weight map corresponding to (b)

Fig. 1: Realization of a Boolean model of fibers and synthetic FIB-SEM image stack generated from it. The SEM imaging plane is $\mathrm{xy}, \mathrm{z}$ is the slicing direction. The xz-view exhibits strong shine through effects.

have been devised to overcome this problem $[7,11,13,14,19,20]$. Nevertheless, being designed for particular structures and SEM modes, they are not generally applicable. Moreover, parameterization requires expert knowledge. Machine learning methods are a popular and already widely used alternative to classical image segmentation. A random forrest is applied to FIB-SEM segmentation in [17]. Convolutional neural networks $(\mathrm{CNN})$ are used with great success also for $3 \mathrm{D}$ image segmentation $[4,15]$. However, in our particular setting of FIB-SEM 
data of highly porous structures, the typical need of these methods for large labeled data is nearly prohibitive. FIB-SEM is rather expensive and manual labeling is difficult to impossible as even the human eye is easily mislead by the shine through artifacts.

Here, we therefore explore the option to train a CNN solely based on synthetic images for which the correct segmentation is readily available. We use a variety of stochastic geometry models $[1,8,10]$ to create porous structures. Digitizations into $3 \mathrm{D}$ images of the respective realizations yield the ground truth for the training phase. The corresponding FIB-SEM stacks are generated based on an analytic representation of the structures - lists of points in space and objects like spheres or cylinders attached to them. These geometries are virtually intersected and SEM images of each planar intersection are simulated as described in [12]. The thus derived FIB-SEM stacks are then used to train the U-net 3D architecture $[4,18]$.

Specially adapted data augmentation and weights as well as the use of versatile structures result in very good segmentations for the synthetic data. Tests on real data are promising, too, results will be reported in [5].

\section{Network architecture and and training the model}

We keep the U-net 3D architecture as specified in [4]. We also follow the original U-net setup [18] in using weighted cross-entropy for measuring similarity of image patches. [18] uses weight maps to assign higher weights to pixels in image regions where objects touch, in order to separate them. We adapt this idea by assigning a higher weight to surface pixels and their neighbors in order to force the network to learn the structure's surface particularly well.

The use of $3 \mathrm{D}$ patches causes them to be small $\left(64^{3}\right.$ pixels) compared to the total image sizes (about $500^{3}-600^{3}$ pixels). Simple tiling results in strong boundary artifacts. To avoid these effects, we therefore apply a sliding window approach with up to 20 pixel wide overlapping regions, depending on local structure size.

In [18], data are augmented excessively by deforming the training images elastically, to force the network to learn invariance to such formations. We combine this approach with brightening and rotating the 3D patches using any of the cube's isometries similar to [4]. To ensure that the training data represent various sizes of the local structures, the patches are chosen with a random crop and resize approach, where the scale factor is variable too.

We trained the network on a Boolean model of fibers (cylinders) [21,22] as, compared to models consisting solely of spheres, the cylinders yield a wider variety of local structures, e. g. both circular and very elongated elliptical crosssections. More precisely, we used a Boolean model of straight cylinders with circular cross-section of diameters uniformly distributed in $60-90 \mathrm{~nm}$ and with lengths uniformly distributed in $300-660 \mathrm{~nm}$. The orientations are uniformly distributed on the upper half-sphere. That is, the model is isotropic. The solid volume fraction is $35 \%$. See Figure 1 for the model realization used. 
From the model realization, the FIB-SEM stack is simulated based on [9] as described in [12]. Throughout, the back scattered electron (BSE) signal is used. The solid component is assumed to be carbon, the primary electrons have an energy of $5 \mathrm{keV}$ as in [12], and the dwelling time is 1 1 s (see e. g. [6] for details on SEM parameters). Both SEM image pixel size and slice distance are $3 \mathrm{~nm}$. The training lasted for 100 epochs, where one epoch equals 50 steps with a batch size of 4 . The initial learning rate of 0.0001 is halved after every 10 epochs.

\section{Results}

The network trained as described in the previous section is now used to segment synthetic FIB-SEM image stacks of a variety of other structures. More precisely, we segment images of Boolean models of spheres and a Cox Boolean model [8] of small spheres nested in large ones (see Figure 2(a)). These are complemented by packings of spheres by the force biased algorithm [2,3], of straight circular cylinders by random sequential adsorption (RSA), and of curved fibers by the Altendorf-Jeulin method (AJ) [1].

We measure the quality of our results by the false negative rate (FNR, the proportion of missed foreground pixels) and the Sørensen-Dice coefficient [23]. The latter is defined as

$$
\operatorname{DICE}(\hat{y}, y)=\frac{2 * \sum_{i=1}^{n} \hat{y}^{i} * y^{i}+\varepsilon}{\sum_{i=1}^{n} \hat{y}^{i}+\sum_{i=1}^{n} y^{i}+\varepsilon}=\frac{2 * T P+\varepsilon}{F N+2 * T P+F P+\varepsilon},
$$

where $n$ is the total number of pixels in the volume, $y$ represents the pixel-wise ground truth, $\hat{y}$ the predicted (segmented) image, and TP, TN, FP, FN are counts of true positive, true negative, false positive and false negative pixels in the prediction, respectively.

Due to the shine through effects described above and the typical coarser sampling in slicing direction, structures reconstructed from FIB-SEM image data tend to be anisotropic to an extent not explainable by the sample production or preparation [14]. All structures considered here are isotropic by design. That is, the distributions of the respective stochastic geometry models are invariant under rotations. Isotropy of the reconstructed structures is therefore a measure for their quality, too. It is by far not an easy task to test the realization of a random closed set for isotropy. Here, we just check the proportion of the mean chord lengths in x- and z-directions $\bar{\ell}_{x}$ and $\bar{\ell}_{z}$ as a rough indicator of artificial anisotropy. The only suspicious case is the RSA cylinder packing. However, here already the $3 \mathrm{D}$ ground truth image has a mean chord length ratio of $\bar{\ell}_{x} / \bar{\ell}_{z}=0.88$.

All results are listed in Table 1 and visualized in Figure 2.

\section{Conclusion}

In this contribution, we show that a deep neural network - namely an adapted U-Net 3D - trained solely on synthetic FIB-SEM image stacks, is capable to reconstruct other highly porous structures from FIB-SEM images. 


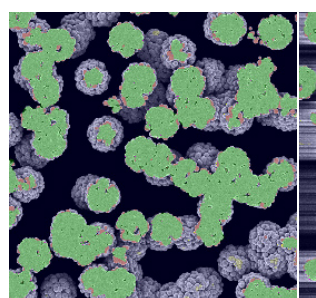

(a) Cox Boolean, xy

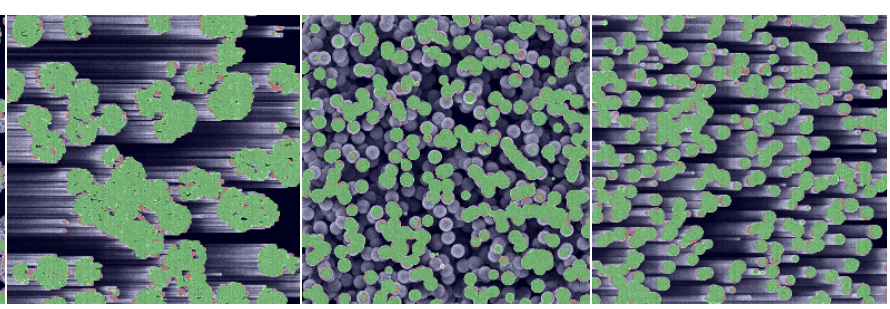

(b) Cox Boolean, xz (c) Boolean spheres, (d) Boolean spheres, xy
$\mathrm{XZ}$

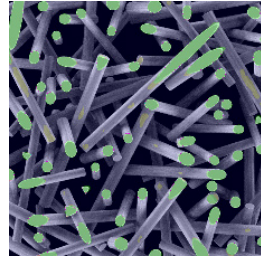

(e) RSA packing of (

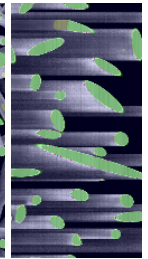

RSA,

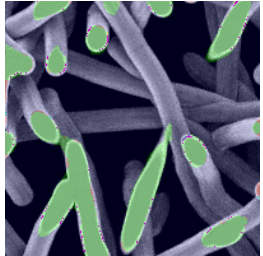

g) AJ fiber packspheres, $x y \quad x z \quad$ ing, $x y$

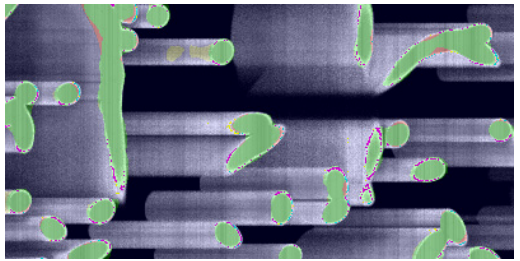

(h) AJ fiber packing, xz

Fig. 2: Segmentation results visualized as overlays on the original FIB-SEM images. Green pixels are correctly segmented foreground pixels (true positive), pixels misclassified as being foreground (false positive) are represented in yellow, and the ones that are misclassified as being background are colored red (false negative).

Table 1: Results of the network trained using solely the Boolean cylinder model as shown in Figure 1.

\begin{tabular}{llccccc}
\hline \multicolumn{7}{c}{ Data } \\
\hline $\begin{array}{l}\text { Boolean } \\
\text { model } \\
\text { of fibers of spheres of cylinders }\end{array}$ & $\begin{array}{c}\text { Boolean } \\
\text { model }\end{array}$ & $\begin{array}{c}\text { RSA } \\
\text { packing }\end{array}$ & $\begin{array}{c}\text { Cox Boolean Altendorf-Jeulin Forced biased } \\
\text { model }\end{array}$ & $\begin{array}{c}\text { fiber } \\
\text { packing }\end{array}$ & $\begin{array}{c}\text { packing } \\
\text { of spheres }\end{array}$ \\
\hline DICE & 0.95 & 0.95 & 0.96 & 0.96 & 0.95 & 0.99 \\
\hline FNR & 0.01 & 0.01 & 0.05 & 0.07 & 0.05 & 0.01 \\
\hline $\bar{\ell}_{x} / \bar{\ell}_{z}$ & 0.98 & 1.02 & 0.80 & 0.94 & 0.99 & 0.91 \\
\hline
\end{tabular}

More details on the stochastic geometry models, wider variation of the FIBSEM imaging parameters, and results on real data will be presented in [5].

The experiments leading to the results presented here highlighted the need for sufficient diversity of local structures in the data used for training. As a cautionary example we show in Figure 3 a result obtained for a cylinder packing using exactly the same network as described above, but trained solely on a Boolean 
model of spheres. Clearly, the thus "mis-trained" net tries to approximate the foreground by spheres.

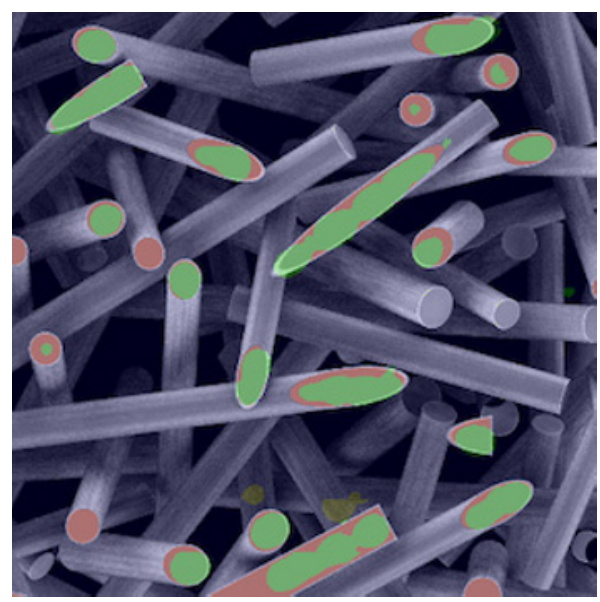

Fig. 3: Overlaid result for RSA fiber packing obtained with net trained on Boolean model of spheres. Meaning of colors as in Figure 2 above.

\section{References}

1. Altendorf, H., Jeulin, D.: Random-walk-based stochastic modeling of threedimensional fiber systems. Phys. Rev. E 83, 041804 (Apr 2011)

2. Bezrukov, A., Bargiet, M., Stoyan, D.: Statistical analysis of simulated random packings of spheres. Part. Part. Systems Char. 19, 111-118 (2002)

3. Bezrukov, A., Stoyan, D., Bargiel, M.: Spatial statistics for simulated packings of spheres. Image Anal. Stereol. 20, 203-206 (2001)

4. Çiçek, Ö., Abdulkadir, A., Lienkamp, S.S., Brox, T., Ronneberger, O.: 3D U-Net: learning dense volumetric segmentation from sparse annotation. In: International Conference on Medical image computing and computer-assisted intervention. pp. 424-432. Springer (2016)

5. Fend, C., Moghiseh, A., Redenbach, C., Schladitz, K.: Reconstruction of highly porous structures from FIB-SEM using a deep neural network trained on synthetic images (2019), in preparation.

6. Goldstein, J., Newbury, D., Joy, D., Lyman, C., Echlin, P., Lifshin, E., Sawyer, L., Michael, J.: Scanning electron microscopy and X-ray microanalysis, 3rd edition. Springer science, New York (2003)

7. Holzer, L., Stenzel, O., Pecho, O., Ott, T., Boiger, G., Gorbar, M., de Hazan, Y., Penner, D., Schneider, I., Cervera, R., Gasser, P.: Fundamental relationships between $3 \mathrm{~d}$ pore topology, electrolyte conduction and flow properties: Towards knowledge-based design of ceramic diaphragms for sensor applications. Materials and Design 99, 314 - 327 (2016) 
8. Jeulin, D.: Morphology and effective properties of multi-scale random sets: A review. Comptes Rendus Mécanique 340(4), 219 - 229 (2012), Recent Advances in Micromechanics of Materials

9. Lowney, J.R.: Monte Carlo simulation of scanning electron microscope signals for lithographic metrology. Scanning 18(4), 301-306 (1996)

10. Ohser, J., Schladitz, K.: 3d Images of Materials Structures - Processing and Analysis. Wiley VCH, Weinheim (2009)

11. Penner, D., Holzer, L.: Characterization and modelling of structure and transport properties of porous ceramics. Publikationen School of Engineering: ZHAW Digital Collection 95(3), E27-E32 (2018)

12. Prill, T., Schladitz, K.: Simulation of FIB-SEM images for analysis of porous microstructures. Scanning 35, 189-195 (2013)

13. Prill, T., Schladitz, K., Jeulin, D., Faessel, M., Wieser, C.: Morphological segmentation of FIB-SEM data of highly porous media. Journal of Microscopy 250(2), 77-87 (2013)

14. Prill, T., Redenbach, C., Roldan, D., Godehardt, M., Schladitz, K., Höhn, S., Sempf, K.: Simulating permeabilities based on 3d image data of a layered nanoporous membrane. International Journal of Solids and Structures (2019)

15. Qi, C.R., Su, H., Mo, K., Guibas, L.J.: Pointnet: Deep learning on point sets for 3d classification and segmentation. In: Proceedings of the IEEE Conference on Computer Vision and Pattern Recognition. pp. 652-660 (2017)

16. Redenbach, C., Schladitz, K., Vecchio, I., Wirjadi, O.: Image analysis for microstructures based on stochastic models. GAMM-Mitteilungen 37(2), 281-305 (2014)

17. Röding, M., Fager, C., Olsson, A., von Corswant, C., Olsson, E., Lorén, N.: Threedimensional reconstruction of microporous polymer films from FIB-SEM nanotomography data using random forests. Microscopy \& Micoranalysis (2019), submitted.

18. Ronneberger, O., Fischer, P., Brox, T.: U-Net: Convolutional networks for biomedical image segmentation. In: International Conference on Medical image computing and computer-assisted intervention. pp. 234-241. Springer (2015)

19. Salzer, M., Prill, T., Spettl, A., Jeulin, D., Schladitz, K., Schmidt, V.: Quantitative comparison of segmentation algorithms for FIB-SEM images of porous media. Journal of Microscopy 257(1), 23-30 (2015)

20. Salzer, M., Thiele, S., Zengerle, R., Schmidt, V.: On the importance of FIB-SEM specific segmentation algorithms for porous media. Materials Characterization $\mathbf{9 5}$, $36-43(2014)$

21. Schneider, R., Weil, W.: Stochastic and Integral Geometry. Probability and Its Applications, Springer, Heidelberg (2008)

22. Stoyan, D., Kendall, W.S., Mecke, J.: Stochastic Geometry and Its Applications. Wiley, Chichester, 2nd edn. (1995)

23. Taha, A.A., Hanbury, A.: Metrics for evaluating 3D medical image segmentation: analysis, selection, and tool. BMC medical imaging 15, 29 (Aug 2015) 
Open Access This chapter is licensed under the terms of the Creative Commons Attribution 4.0 International License (http://creativecommons.org/licenses/by/4.0/), which permits use, sharing, adaptation, distribution and reproduction in any medium or format, as long as you give appropriate credit to the original author(s) and the source, provide a link to the Creative Commons license and indicate if changes were made.

The images or other third party material in this chapter are included in the chapter's Creative Commons license, unless indicated otherwise in a credit line to the material. If material is not included in the chapter's Creative Commons license and your intended use is not permitted by statutory regulation or exceeds the permitted use, you will need to obtain permission directly from the copyright holder.

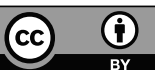

